\title{
Generalized Duality for a Nondifferentiable Control Problem
}

\author{
I. Husain ${ }^{1, *}$, Vikas K. Jain ${ }^{1}$, Abdul Raoof Shah ${ }^{2}$ \\ ${ }^{1}$ Department of Mathematics, Jaypee University of Engineering and Technology, Guna, India \\ ${ }^{2}$ Department of Statistics, University of Kashmir, Srinagar, India \\ *Corresponding author: ihusain11@yahoo.com, jainvikas13@yahoo.com
}

Received March 13, 2014; Revised May 20, 2014; Accepted June 30, 2014

\begin{abstract}
A generalized dual to a control problem containing support functions is formulated and various duality theorems are established under generalized convexity hypotheses. This dual model represents the combination of Wolfe and Mond-Weir type dual models to the control problem and hence it is described as a generalized dual. Some special cases are obtained. A close relationship of duality results with those of the nonlinear programming problems involving support functions is indicated.
\end{abstract}

Keywords: control problem, support function, optimality conditions, generalized duality, converse duality, nonlinear programming

Cite This Article: I. Husain, Vikas K. Jain, and Abdul Raoof Shah, "Generalized Duality for a Nondifferentiable Control Problem." American Journal of Applied Mathematics and Statistics, vol. 2, no. 4 (2014): 193-202. doi: 10.12691/ajams-2-4-3.

\section{Introduction}

In [3], Husain et al. considered the following control problem containing support functions:

$$
\text { (CP): } \underset{(x, u)}{\operatorname{Minimize}} \int_{I}(f(t, x, u)+S(u(t) \mid K)) d t
$$

subject to

$$
\begin{gathered}
x(a)=0, x(b)=0, \\
\dot{x}(t)=h(t, x, u), t \in I \\
g^{i}(t, x, u)+S\left(x(t) \mid C^{i}\right) \leq 0, \quad i=1,2, \ldots . m, t \in I
\end{gathered}
$$

where

(i) $x: I \rightarrow R^{n}$ is a differentiable state vector function with its derivative $\dot{x}$ and $\mathrm{u}: I \rightarrow R^{m}$ is a smooth control vector function,

(ii) $R^{n}$ denotes an n-dimensional Euclidean space and $I=[a, b]$ is a real interval.

(iii) $\quad f: I \times R^{n} \times R^{m} \rightarrow R, \mathrm{~g}^{i}: I \times R^{n} \times R^{m} \rightarrow R, i=1,2, \ldots . m$ and $\mathrm{h}: I \times R^{n} \times R^{m} \rightarrow R^{n}$ are continuously differentiable.

(iv) $s(x(t) \mid \mathrm{K})$ and $s\left(x(t) \mid \mathrm{C}^{j}\right), \mathrm{j}=1,2, \ldots, \mathrm{m}$. are the support functions of the compact sets $K$ and $\mathrm{C}^{j}(j=1,2, \ldots, m)$ respectively.

Denote the partial derivatives of $f$ where by $f_{t}, f_{x}$ and $f_{y}$,

$$
\begin{aligned}
& f_{t}=\frac{\partial f}{\partial t}, f_{x}=\left[\frac{\partial f}{\partial x^{1}}, \frac{\partial f}{\partial x^{2}}, \ldots, \frac{\partial f}{\partial x^{n}}\right], \\
& f_{u}=\left[\frac{\partial f}{\partial u^{1}}, \frac{\partial f}{\partial u^{2}}, \ldots, \frac{\partial f}{\partial u^{m}}\right],
\end{aligned}
$$

where superscripts denote the vector components. Similarly we have $h_{t}, h_{x}, h_{u}$ and $g_{t}, g_{x}, g_{u}$. Designate by $X$. The space of continuously differentiable state functions $x: I \rightarrow R^{n}$ such that $x(a)=0$ and $x(b)=0$ and is equipped with the norm $\|x\|=\|x\|_{\infty}+\left\|D_{x}\right\|_{\infty}$, and $U$, the space of piecewise continuous control vector functions $\mathrm{u}: I \rightarrow R^{m}$ having the uniform norm \|\|$_{\infty}$. The differential equation (2) with initial conditions expressed as $x(t)=x(a)+\int_{a}^{b} h(s, x(s), u(s)) d s, t \in I$ may be written as $H=H(x, u)$, where $H: X \times U \rightarrow C\left(I, R^{n}\right), C\left(I, R^{n}\right)$ being the space of continuous function from $I$ to $R^{n}$ defined as $H(x, u)(t)=h(t, x(t), u(t))$. In the derivation of the optimality conditions, some constraint qualification to make the equality constraints locally solvable is needed. For this the Fre'che't derivative of $D_{x}-H(x, u)=Q(x, u)$, (say) with respect to $(x, u)$, namely $Q^{\prime}=Q^{\prime}(x, u)=\left[D-H_{x}(x, u),-H_{u}(x, u)\right]$ are required to be surjective. Husain et al. [3] established the following Fritz type necessary conditions for the control problem (CP): 
Proposition 1. (Fritz John Necessary Conditions): If $(\bar{x}, \bar{u})$ is an optimal solution of (CP) and the Fre'che't derivative $Q^{\prime}$ is surjective, then there exists Langrange multipliers $\alpha \in R$ and piecewise smooth $\lambda: I \rightarrow R^{m}$, $u: I \rightarrow R^{r}, z: I \rightarrow R^{m}$ and $\omega^{j}: I \rightarrow R^{n}$ such that for all $t$,

$$
\begin{gathered}
\alpha f_{x}(t, \bar{x}, \bar{u})+\sum_{j=1}^{m} \lambda^{j}(t)\left(g_{x}^{j}(t, \bar{x}, \bar{u})+\omega^{j}(t)\right) \\
+\mu(t)^{T} h_{x}(t, \bar{x}, \bar{u})=\dot{\mu}(t), t \in I \\
\alpha f_{u}(t, \bar{x}, \bar{u})+\lambda^{T}(t) g_{u}(t, \bar{x}, \bar{u}) \\
+\mu(t)^{T} h_{u}(t, \bar{x}, \bar{u})=0, t \in I \\
\sum_{j=1}^{m} \lambda^{j}(t)\left(g_{x}^{j}(t, \bar{x}, \bar{u})+x^{T}(t) \omega^{j}(t)\right)=0, t \in I \\
\mu^{T}(t) z(t)=S(x(t) \mid K) \\
x(t) \omega^{j}(t)=S\left(x(t) \mid C^{j}\right), j=1,2, \ldots, m \\
z(t) \in K, \omega^{j}(t) \in C^{j}, j=1,2, \ldots, m \\
(\alpha, \lambda(t)) \geq 0, t \in I \\
(\alpha, \lambda(t), u(t)) \neq 0, t \in I
\end{gathered}
$$

As in [5], Husain et al. [3] pointed out if the optimal solution for (CP) is normal, then the Fritz John type optimal conditions reduce to the following Karush-KuhnTucker optimal conditions:

Proposition 2. If $(\bar{x}, \bar{u})$ is an optimal solution and is normal and $Q^{\prime}$ is surjective, there exist piecewise smooth $\lambda: I \rightarrow R^{m} \quad$ with $\quad \lambda^{T}=\left(\lambda_{1}, \lambda_{2}, \ldots, \lambda_{m}\right), u: I \rightarrow R^{n}$, $\mathrm{z}: I \rightarrow R^{n}$ and $\omega^{j}: I \rightarrow R^{n}, j=1,2, \ldots, m$ such that

$$
\begin{gathered}
f_{x}(t, \bar{x}, \bar{u})+\sum_{j=1}^{m} \lambda^{j}(t)\left(g_{x}^{j}(t, \bar{x}, \bar{u})+\omega^{j}(t)\right) \\
+\mu(t)^{T} h_{x}(t, \bar{x}, \bar{u})=\dot{\mu}(t) \\
f_{u}(t, \bar{x}, \bar{u})+\lambda^{T}(t) g_{u}(t, \bar{x}, \bar{u}) \\
+\mu(t)^{T} h_{u}(t, \bar{x}, \bar{u})=0, t \in I \\
\sum_{j=1}^{m} \lambda^{j}(t)\left(g_{x}^{j}(t, \bar{x}, \bar{u})+x^{T}(t) \omega^{j}(t)\right)=0, t \in I \\
u(t)^{T} z(t)=S(x(t) \mid K) \\
x(t)^{T} \omega^{j}(t)=S\left(x(t) \mid C^{j}\right), j=1,2, \ldots, m \\
\lambda^{i}(t) \geq 0, t \in I, j=1,2, \ldots, m . \\
z(t) \in K, \omega^{j}(t) \in C^{j}, j=1,2, \ldots, m .
\end{gathered}
$$

Using the Karush-Kuhn-Tucker type necessary optimality conditions, Husain et al. [3] constructed the following Wolf type dual control problem to (CP) and proved various duality results:

(WCD): Maximize $\int_{I}\left\{\begin{array}{l}f(t, x, u)+\mu(t)^{T} z(t) \\ +\sum_{j=1}^{m} \lambda^{i}(t)\left(\begin{array}{l}g^{j}(t, x, u) \\ +x(t)^{T} \omega^{j}(t)\end{array}\right) \\ +\mu(t)^{T}(h(t, x, u)-\dot{x}(t))\end{array}\right\} d t$

subject to

$$
\begin{gathered}
x(a)=0, \alpha(b)=0 \\
f_{x}(t, x, u)+\sum_{j=1}^{m} \lambda^{i}(t)\left(g^{j}(t, x, u)+\omega^{j}(t)\right) \\
+\mu(t)^{T} h_{x}-\dot{\mu}(t)=0, t \in I \\
f_{u}(t, x, u)+\lambda^{T}(t) g(t, x, u)+\mu(t)^{T} h_{u}=0, t \in I \\
\lambda^{i}(t) \geq 0, t \in I, i=1,2, \ldots, m \\
z(t) \in K, \omega^{i}(t) \in C^{i}, i=1,2, \ldots, m
\end{gathered}
$$

The problem (WCD) is a dual to (CP) assuming that $\int_{I}\left\{f+\sum_{j=1}^{m} \lambda^{i}(t)\left(g^{j}(.,)+.(.)^{T}().\right)+\mu^{T}(t)(h-\dot{x}(t))\right\} d t$ is pseudo convex in $(x, u)$ for all $z(t) \in R^{m}$ and $\omega^{j}(t) \in R^{n}, j=1,2, \ldots, m$.

Husain et al. [4] further weakened the generalized convexity for duality by constructing a Mond-Weir type dual to (CP) given below.

(M-WCD): Maximize $\int_{I}\left\{f(t, x, u)+u^{T}(t) z(t)\right\} d t$ subject to

$$
\begin{gathered}
x(a)=0=x(b) \\
f_{x}(t, x, u)+\sum_{j=1}^{m} \lambda^{j}(t)\left(g_{x}^{j}(t, x, u)+x(t)^{T} \omega^{i}(t)\right) \\
+\mu(t)^{T} h_{x}(t, x, u)=\dot{\mu}(t), t \in I \\
f_{u}(t, x, u)+z+\lambda^{t} g_{u}(t, x, u)+\mu(t)^{T} h_{u}(t, x, u)=0, t \in I \\
\sum_{j=1}^{m} \lambda^{i} \int_{I}\left\{g^{j}(t, x, u)+x(t)^{T} \omega^{j}\right\} d t \geq 0 \\
\int_{I} \mu(t)^{T}(h(t, x, u)-\dot{x}) d t \geq 0 \\
\lambda(t) \geq 0, t \in I \\
z(t) \in K \\
\omega^{j}(t) \in C^{j}, j=1,2, \ldots, m
\end{gathered}
$$

where

(i) $K$ and $C^{j}, j=1,2, \ldots, m$ are compact sets in $R^{n}$, and (ii) $\lambda: I \rightarrow R^{m}, \omega: I \rightarrow R^{n}$ and $\mu: I \rightarrow R^{n}$ are piecewise smooth functions and 
Husain et al. [4] proved duality theorems for the problem (CP) and (M-WCD) under the assumptions of pseudoconvexity of $\int_{I}\{f(t, \cdot, \cdot)+(\cdot) z(t)\} d t$ for $z(t) \in K$ and quasi convexity of $\sum_{j=1}^{m} \int_{I} \lambda^{i}(t)\left(g^{j}(t, \cdot, \cdot)+(\cdot)^{T} \omega^{j}(t)\right) d t$ and

$$
\int_{I} \mu^{T}(t)(h(t, \cdot, \cdot)-x(\cdot)) d t \quad \text { for }
$$

all $\omega^{j}(t) \in C^{j}, j=1,2, \ldots, m$.

We review some well known facts about a support function for easy reference. Let $\Gamma$ be a compact convex set in $R^{n}$. Then the support function of $\Gamma$ denoted by $S(x(t) \mid \Gamma)$ is defined as

$$
S(x(t) \mid \Gamma)=\max \left\{x(t)^{T} v(t): v(t) \in \Gamma, t \in I\right\}
$$

A support function, being convex and everywhere finite, has a subdifferential in the sense of convex analysis, that is, there exists $\mathrm{z}$ such that $S(y(t) \mid \Gamma) \geq S(x(t) \mid \Gamma)+z(t)^{T}(y(t)-x(t)) \quad$ for $\quad$ all $x$. The subdifferential of $S(x(t) \mid \Gamma)$ is given by $S(x(t) \mid \Gamma)=\left\{z(t) \in \Gamma: z(t)^{T} x(t)=S(x(t) \mid \Gamma)\right\} . \quad$ Let $N_{\Gamma}(x(t))$ be normal cone at a point $x(t) \in \Gamma$. Then $y(t) \in N_{\Gamma}(x)$ if and only if $S(y(t) \mid \Gamma)=x(t)^{T} y(t)$ or equivalently, $x(t)$ is in the subdifferential of $s$ at $y(t)$.

In this paper, we propose a generalized dual to (CP) and prove various duality theorems under appropriate generalized convexity assumption. From our duality results, special cases are deduced and it is shown that our results derived in this research can be considered as dynamic generalization of those of nonlinear programming problems having support functions.

\section{Generalized Duality}

Let $M=\{1,2, \ldots, m\}, N=\{1,2, \ldots, n\}, I_{\alpha} \subseteq M, \alpha=0,1, \ldots r$ with $I_{\alpha} \cap I_{\beta}=\phi, \alpha \neq \beta$, and $\bigcup_{\alpha=0}^{r} I_{\alpha}=M$, and $J_{\alpha} \subseteq N$, $\alpha=0,1, \ldots r$ with $J_{\alpha} \cap J_{\beta}=\phi, \alpha \neq \beta$, and $\bigcup_{\alpha=0}^{r} J_{\alpha}=N$.

We propose the following generalized dual to the problem (CP) and prove various duality theorem under appropriate generalized convexity condition:

(GCD): Maximize $\int_{I}\left(\begin{array}{l}f(t, x, u)+u(t)^{T} z(t) \\ +\sum_{i \in I_{0}} \lambda^{i}(t)\left(\begin{array}{l}g^{i}(t, x, u) \\ +x(t)^{T} \omega^{i}(t)\end{array}\right) \\ +\sum_{j \in j_{0}} \mu^{j}(t)\left(h^{j}(t, x, u)-\dot{x}^{j}\right)\end{array}\right) d t$

subject to

$$
\begin{gathered}
x(a)=0, x(b)=0 \\
f_{x}(t, x, u)+\sum_{i=1}^{m} \lambda^{i}(t)\left(g_{x}{ }^{i}(t, x, u)+\omega^{i}(t)\right) \\
+\dot{\mu}(t) h_{x}(t, x, u)+\dot{\mu}(t)=0, t \in I \\
f_{u}(t, x, u)+z(t)+\lambda^{T}(t) g_{u}(t, x, u) \\
+\mu(t)^{T} h_{u}(t, x, u)=0, t \in I
\end{gathered}
$$

$$
\begin{gathered}
\int \sum_{I} \lambda_{i \in I_{\alpha}}(t)\left(g^{i}(t, x, u)+u(t)^{T} \omega^{i}(t)\right) d t \geq 0, \alpha=1,2, \ldots, r(14) \\
\int_{I} \sum_{j \in J_{\alpha}} \mu^{i}(t)\left(h^{j}(t, x, u)-x^{j}(t)\right) d t \geq 0, \alpha=1,2, \ldots, r(15) \\
\lambda^{i}(t) \geq 0, i=1,2, \ldots, m \\
z(t) \in K, \omega^{i}(t) \in C^{i}, i=1,2, \ldots, m
\end{gathered}
$$

Theorem 1 (Weak duality): let $(\bar{x}, \bar{u})$ be feasible for (CP) and $\left(x, u, \lambda, z, \mu, \omega^{1}, \ldots, \omega^{m}\right)$ with $\lambda^{T}=\left(\lambda^{1}, \ldots, \lambda^{m}\right)$ and $\mu^{T}=\left(\mu^{1}, \ldots, \mu^{m}\right)$ feasible for (GCD). If for all feasible $\left(\bar{x}, \bar{u}, \mu, x, u, \lambda, z, \omega^{1}, \ldots, \omega^{m}\right)$,

$$
\int_{I}\left\{\begin{array}{l}
f(t, . . .)+(.)^{T} z(t) \\
+\sum_{i \in I_{0}} \lambda^{i}(t)\left(g^{i}(t, . . .)+(.)^{T} \omega^{i}(t)\right) \\
+\sum_{j \in J_{0}} \mu^{j}(t)\left(h^{j}(t, . . .)-\dot{x}^{j}(.)\right)
\end{array}\right\} d t
$$

is pseudoconvex, and $\int_{I} \sum_{i \in I_{\alpha}}\left(\lambda^{i}(t)\left(\begin{array}{l}g^{i}(t, ., .) \\ +(.)^{T} \omega^{i}(t)\end{array}\right)\right) d t$, and $\int_{I} \sum_{j \in J_{\alpha}}\left(\mu^{j}(t)\left(h^{j}(t, . .)-.\dot{x}^{j}().\right)\right) d t, \quad \alpha=1,2, \ldots, r \quad$ are quasiconvex, then

$$
\inf (C P) \geq \operatorname{Sup}(G C D) \text {. }
$$

Proof: Since $(\bar{x}, \bar{u})$ is feasible for (CP) and $\left(x, u, \lambda, z, \mu, \omega^{1}, \ldots, \omega^{m}\right)$ is feasible for (GCD), we have

$$
\begin{aligned}
& \int_{I} \sum_{i \in I_{\alpha}} \lambda^{i}(t)\left(g^{i}(t, \bar{x}, \bar{u})+\bar{x}(t)^{T} \omega^{i}(t)\right) d t \\
& \leq \int_{I} \sum_{i \in I_{\alpha}} \lambda^{i}(t)\left(g(\bar{x}, \bar{u})+x(t)^{T} \omega^{i}(t)\right) d t, \alpha=1,2, \ldots, r
\end{aligned}
$$

and

$$
\begin{aligned}
& \int_{I} \sum_{j \in J_{\alpha}} \mu^{j}(t)\left(h^{j}(t, \bar{x}, \bar{u})-\dot{\bar{x}}^{j}(t)\right) d t \\
& \leq \int_{I} \sum_{j \in J_{\alpha}} \mu^{j}(t)\left(h^{j}(t, x, u)-\dot{x}^{j}(t)\right) d t, \alpha=1,2, \ldots, r .
\end{aligned}
$$


By the quasiconvexity of $\sum_{i \in I_{\alpha} I} \lambda^{i}(t)\left(\begin{array}{l}g^{i}(t, x, u) \\ +u(t)^{T} \omega^{i}(t)\end{array}\right) d t$ and $\sum_{j \in J_{\alpha} I} \int_{I} \mu^{j}(t)\left(h^{j}(t, x, u)-\dot{x}^{j}(t)\right) d t, \quad \alpha=1,2, \ldots, r$, the above inequality respectively yields,

$$
\sum_{i \in I_{\alpha} I} \int_{I} \lambda^{i}(t)\left\{\begin{array}{l}
(\bar{x}-x)^{T}\left(g_{x}{ }^{i}(t, x, u)+\omega^{i}(t)\right) \\
+(\bar{u}-u)^{T} g_{u}{ }^{i}(t, x, u)
\end{array}\right\} d t \leq 0
$$

and

$$
\sum_{j \in J} \int_{\alpha}\left\{\begin{array}{l}
(\bar{x}-x)^{T}\left(\mu^{j}(t) h_{x}^{j}(t, x, u)-\dot{\mu}^{j}(t)\right) \\
+(\bar{u}-u)^{T} \mu^{j}(t) h_{u}^{j}(t, x, u)
\end{array}\right\} d t \leq 0
$$

Hence

$$
\sum_{i \in M-I_{0} I} \int \lambda^{i}(t)\left\{\begin{array}{l}
\left.(\bar{x}-x)^{T}\left(g_{x}{ }^{i}(t, x, u)+\omega^{i}(t)\right)\right) \\
+(\bar{u}-u)^{T} g_{u}{ }^{i}(t, x, u)
\end{array}\right\} d t \leq 0
$$

and

$$
\sum_{j \in N-J_{0}} \int_{I}\left\{\begin{array}{l}
(\bar{x}-x)^{T}\left(\mu^{j}(t) h_{x}^{j}(t, x, u)+\dot{\mu}^{j}(t)\right) \\
+(\bar{u}-u)^{T} \mu^{j}(t) h_{u}^{j}(t, x, u)
\end{array}\right\} d t \leq 0 .
$$

Combining the above inequalities and the using equality constraints (12) and (13), we have

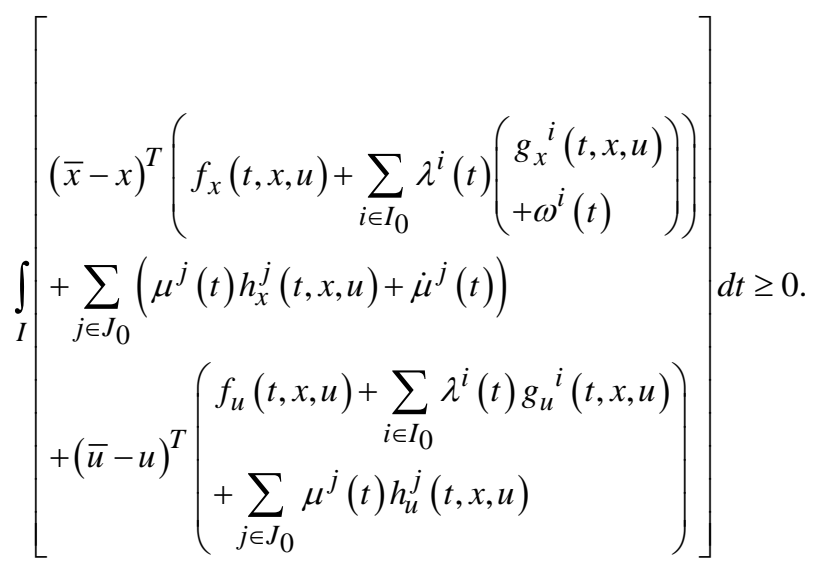

This, because of pseudoconvexity of

$$
\int_{I}\left\{\begin{array}{l}
f(t, . . .)+(.)^{T} z(t)+\sum_{i \in I_{0}} \lambda^{i}(t)\left(\begin{array}{l}
g^{i}(t, . . .) \\
+(.)^{T} \omega^{i}(t)
\end{array}\right) \\
+\sum_{j \in J_{0}} \mu^{j}(t)\left(h^{j}(t, . . .)-\dot{x}^{j}(.)\right)
\end{array}\right\} d t
$$

at $(\bar{x}, \bar{u})$, we have

$$
\int_{I}\left\{\begin{array}{l}
f(t, \bar{x}, \bar{u})+\bar{u}^{T}(t) z(t)+\sum_{i \in I_{0}} \lambda^{i}(t)\left(\begin{array}{l}
g^{i}(t, \bar{x}, \bar{u}) \\
+\bar{x}(t)^{T} \omega^{i}(t)
\end{array}\right) \\
+\sum_{j \in J_{0}} \mu^{j}(t)\left(h^{j}(t, \bar{x}, \bar{u})-\dot{\bar{x}}^{j}(t)\right)
\end{array}\right\} d t
$$

$$
\geq \int_{I}\left\{\begin{array}{l}
f(t, x, u)+u^{T}(t) z(t)+\sum_{i \in I_{0}} \lambda^{i}(t)\left(\begin{array}{l}
g^{i}(t, x, u) \\
+x(t)^{T} \omega^{i}(t)
\end{array}\right) \\
+\sum_{j \in J_{0}} \mu^{j}(t)\left(h^{j}(t, x, u)-\dot{x}^{j}(t)\right)
\end{array}\right\} d t .
$$

Using $\quad \bar{u}(t)^{T} z(t) \leq S(\bar{u}(t) \mid K) \quad$ and $\bar{x}(t)^{T} \omega^{j}(t) \leq S\left(\bar{x}(t) \mid C^{j}\right), \quad j=1,2, \ldots, m$, together with feasibility of $(\bar{x}, \bar{u})$ for (CP) in the above inequality, we have

$$
\begin{aligned}
& \int_{I}\{f(t, \bar{x}, \bar{u})+S(\bar{u}(t) \mid K)\} d t \\
& \geq \int_{I}\left\{\begin{array}{l}
\left.f(t, x, u)+u^{T}(t) z(t)+\sum_{i \in I_{0}} \lambda^{i}(t)\left(\begin{array}{l}
g^{i}(t, x, u) \\
+x(t)^{T} \omega^{i}(t)
\end{array}\right)\right\} d t \\
+\sum_{j \in J_{0}} \mu^{j}(t)\left(h^{j}(t, x, u)-\dot{x}^{j}(t)\right)
\end{array}\right.
\end{aligned}
$$

yielding

$$
\inf (C P) \geq \operatorname{Sup}(G C D) .
$$

Theorem 2 (Strong Duality): if $(\bar{x}, \bar{u})$ is an optimal solution of (CP) and is normal , then there exist piecewise smooth $z: I \rightarrow R^{n}, \mu: I \rightarrow R^{n}, \lambda^{i}: I \rightarrow R, i=1,2, \ldots, m$ and $\quad \omega^{j}: I \rightarrow R^{n}, \quad j=1,2, \ldots, m \quad$ such that $\left(\bar{x}, \bar{u}, \bar{z}, \lambda^{1}, \ldots, \lambda^{m}, \omega^{1}, \ldots, \omega^{m}, \mu\right)$ is feasible for (GCD), and the corresponding values of (CP) and (GCD) are equal. If the hypotheses of Theorem 1 hold, then $\left(\bar{x}, \bar{u}, \bar{z}, \lambda^{1}, \ldots, \lambda^{m}, \omega^{1}, \ldots, \omega^{m}, \mu\right)$ is an optimal solution of (GCD).

Proof: Since $(\bar{x}, \bar{u})$ is an optimal solution of (CP) and is normal , then from Proposition 2, there exist piecewise smooth $\quad z: I \rightarrow R^{n}, \quad \lambda^{j}: I \rightarrow R, \quad j=1,2, \ldots, m$, $\omega^{j}: I \rightarrow R^{n}, \quad j=1,2, \ldots, n$ and $\mu: I \rightarrow R^{n}$ such that conditions (4)-(10) hold. So $\left(\bar{x}, \bar{u}, \bar{z}, \lambda^{1}, \ldots, \lambda^{m}, \omega^{1}, \ldots, \omega^{m}, \mu\right)$ is feasible for (GCD) and in view of conditions (4), (5), (6), (9) and (10), the equality of the objective functionals follows. If $\int_{I}\left(\begin{array}{l}f(t, . . .)+(.)^{T} z+\sum_{i \in I_{0}} \lambda^{i}(t)\left(g^{i}(t, . . .)+(.)^{T} \omega^{i}(t)\right) \\ +\sum_{j \in j_{0}} \mu^{j}(t)\left(h^{j}(t, . . .)+\dot{x}^{j}(.)\right)\end{array}\right) d t$ is pseudoconvex, and $\sum_{i \in I_{0}} \int_{I} \lambda^{i}(t)\left(g^{i}(t, . .)+.(.)^{T} \omega^{j}(t)\right) d t$ and $\sum_{j \in j_{0}} \int_{I} \mu^{j}(t)\left(h^{j}(t, .)-.\dot{x}^{j}(t)\right) d t$ are quasiconvex for all $z(t) \in K$ and $\omega^{j}(t) \in C^{j}, j=1,2, . ., m$, then from Theorem $1\left(\bar{x}, \bar{u}, \bar{z}, \lambda^{1}, \ldots, \lambda^{m}, \omega^{1}, \ldots, \omega^{m}, \mu\right)$ must be an optimal solution of (GCD). 
Theorem 3 (Strict converse duality): Let the problem (CP) have an optimal solution $(\bar{x}, \bar{u})$ that satisfies the normality condition and $\left(\hat{x}, \hat{u}, \hat{\lambda}^{1}, \ldots, \hat{\lambda}^{m}, \hat{z}, \hat{\mu}, \hat{\omega}^{1}, \ldots, \hat{\omega}^{m}\right)$ be optimal solution of (GCD) if

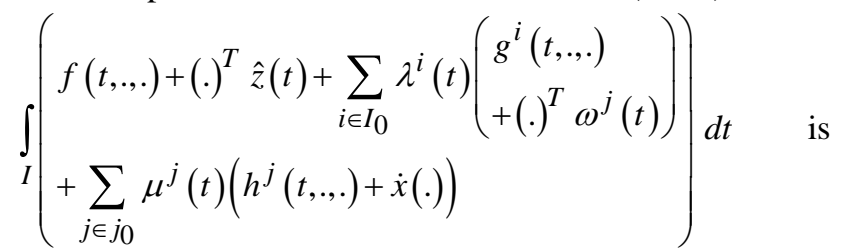
strictly pseudoconvex, and $\int_{I}\left\{\sum_{i \in I_{0}} \lambda^{i}(t)\left(\begin{array}{l}g^{i}(t, . . .) \\ +(.)^{T} \omega^{j}(t)\end{array}\right)\right\} d t$ and $\int_{I}\left\{\sum_{j \in j_{0}} \mu^{j}(t)\left(h^{j}(t, . .)+.\dot{x}^{j}().\right)\right\} d t$ are quasi convex for all $z \in K, \omega^{j} \in C^{j}, j=1,2, \ldots, m$, then $(\bar{x}, \bar{u})=(\hat{x}, \hat{u})$, i.e., $(\hat{x}, \hat{u})$ is an optimal solution of (CP).

Proof: We shall assume that $(\bar{x}, \bar{u}) \neq(\hat{x}, \hat{u})$ and exhibit a contradiction, since $(\bar{x}, \bar{u})$ is an optimal solution of (CP), it follows from Theorem 2 there exist $\bar{\lambda}^{i}: I \rightarrow R,(i=1,2, \ldots, m), \bar{\omega}^{j}: I \rightarrow R^{n},(j=1,2, \ldots, m)$ and $\quad \mu^{j}: I \rightarrow R^{n},(j=1,2, \ldots, m)$, such that $\left(\bar{x}, \bar{u}, \bar{z}, \bar{\lambda}^{1}, \ldots, \bar{\lambda}^{m}, \bar{\mu}^{1}, \ldots, \bar{\mu}^{n}, \bar{\omega}^{1}, \ldots, \bar{\omega}^{n}\right) \quad$ is $\quad$ an optimal solution of (GCD). Hence

$$
\begin{aligned}
& \int_{I}\left\{\begin{array}{l}
f(t, \bar{x}, \bar{u})+\bar{u}^{T} \bar{z}+\sum_{i \in I_{0}} \bar{\lambda}^{i}\left(g^{i}(t, \bar{x}, \bar{u})+\bar{\omega}^{i}(t)\right) \\
+\sum_{j \in J_{0}} \bar{\mu}^{j}(t)\left(h^{j}(t, \bar{x}, \bar{u})-\dot{\bar{x}}^{j}\right)
\end{array}\right\} d t \\
& =\int_{I}\left\{\begin{array}{l}
f(t, \hat{x}, \hat{u})+\hat{u}^{T} \hat{z}+\sum_{i \in I_{0}} \hat{\lambda}^{i}\left(g^{i}(t, \hat{x}, \hat{u})+\hat{\omega}^{i}(t)\right) \\
+\sum_{j \in J_{0}} \hat{\mu}^{j}(t)\left(h^{j}(t, \hat{x}, \hat{u})-\dot{\hat{x}}\right)
\end{array}\right\} d t .
\end{aligned}
$$

together with the feasibility of $(\bar{x}, \bar{u})$ for (CP) and $\left(\hat{x}, \hat{u}, \hat{\lambda}^{1}, \ldots, \hat{\lambda}^{m}, \hat{z}, \hat{\mu}, \hat{\omega}^{1}, \ldots, \hat{\omega}^{m}\right) \quad$ for $\quad$ (GCD). $\bar{x}(t)^{T} \bar{\omega}^{j}(t)=S\left(\bar{x}(t) \mid C^{j}\right)$, for $\bar{\omega}^{j} \in C^{j}, j=1,2, \ldots, m$, we have

$$
\begin{aligned}
& \sum_{i \in I_{\alpha} I} \int_{\hat{\lambda}^{i}}\left(\left(g^{i}(t, \bar{x}, \bar{u})+\bar{x}(t)^{T} \bar{\omega}^{i}(t)\right)\right) d t \\
& \leq \sum_{j \in J_{\alpha} I} \int_{I} \hat{\lambda}^{j}\left(\left(g^{j}(t, \hat{x}, \hat{u})+\hat{x}(t)^{T} \hat{\omega}^{i}(t)\right)\right) d t
\end{aligned}
$$

Also

$$
\begin{aligned}
& \sum_{j \in J_{\alpha}} \int_{I} \hat{\mu}^{j}(t)\left(h^{j}(t, \bar{x}, \bar{u})-\bar{x}^{j}(t)\right) d t \\
\leq & \sum_{j \in J_{\alpha} I} \int_{\mu^{j}}(t)\left(h^{j}(t, \hat{x}, \hat{u})-\dot{\hat{x}}^{j} \hat{\omega}\right) d t, \alpha=1,2, \ldots, r
\end{aligned}
$$

These, because of quasiconvexity hypothesis and merging their implication and then using equality constraints of (GCD), we have

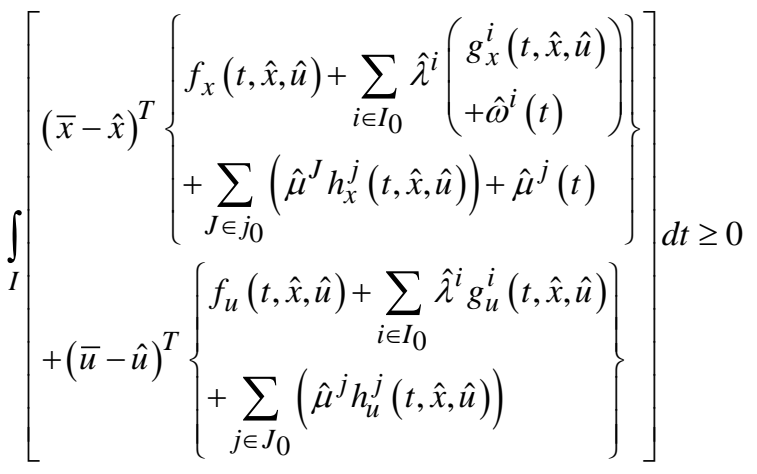

This, in view of the strict pseudoconvexity of

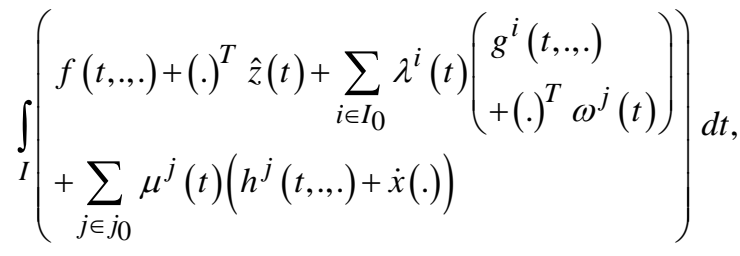

implies

$$
\begin{aligned}
& \int_{I}\left\{\begin{array}{l}
f(t, \bar{x}, \bar{u})+\bar{u} \hat{z}+\sum_{i \in I_{0}} \hat{\lambda}^{i}\left(g^{i}(t, \bar{x}, \bar{u})+\dot{\bar{x}}^{T} \omega^{i}\right) \\
+\sum_{j \in J_{0}} \hat{\mu}^{j}(t)\left(h^{j}(t, \bar{x}, \bar{u})+\dot{\bar{x}}^{j}\right)
\end{array}\right\} d t \\
& \geq \int_{I}\left\{\begin{array}{l}
\left.f(t, \hat{x}, \hat{u})+\sum_{i \in I_{0}} \hat{\lambda}^{i}\left(g^{j}(t, \hat{x}, \hat{u})+\hat{x}^{T} \hat{\omega}^{i}\right)\right\} \\
+\sum_{j \in J_{0}} \hat{\mu}^{j}(t)\left(h^{j}(t, \hat{x}, \hat{u})+\dot{\hat{x}}^{j}\right)
\end{array}\right\} d t \\
& =\int_{I}\left\{\begin{array}{l}
f(t, \bar{x}, \bar{u})+\bar{x}^{T} \bar{z}+\sum_{i \in I_{0}} \bar{\lambda}^{i}\left(g^{i}(t, \bar{x}, \bar{u})+\bar{x}^{T} \omega^{i}\right) \\
+\sum_{j \in J_{0}} \bar{\mu}^{j}(t)\left(h^{j}(t, \bar{x}, \bar{u})+\dot{\bar{x}}^{j}\right)
\end{array}\right\} d t .
\end{aligned}
$$

This in view of (2) and (3), yields.

$$
\begin{aligned}
& \int_{I}\left(f(t, \bar{x}, \bar{u})+\bar{u}(t)^{T} \hat{z}(t)\right) d t \\
& >\int_{I}\left(f(t, \bar{x}, \bar{u})+\bar{u}(t)^{T} \bar{z}(t)\right) d t,
\end{aligned}
$$

which gives

$$
\int_{I} \bar{u}^{T}(t) \hat{z}(t) d t>\int_{I} \bar{u}^{T} \bar{z}(t) d t .
$$

In view of $\quad \bar{u}^{T} \hat{z}(t) \leq S(\bar{u}(t) \mid K) \quad$ and $\bar{u}^{T}(t) \bar{z}(t) \leq S(\bar{u}(t) \mid K)$, this implies

$$
\int_{I} S(\bar{u}(t) \mid K) d t>\int_{I} S(\bar{u}(t) \mid K) d t,
$$

which is absurd. Hence $(\bar{x}, \bar{u})=(\hat{x}, \hat{u})$.

\section{Converse Duality}


In this section, we shall prove the converse duality under the assumption $f, g$ and $h$ are twice continuously differentiable. The problem (GCD) may be written in the following form:

Maximize $\psi\left(t, x, u, z, \lambda^{1}, \ldots, \lambda^{m}, \mu^{1}, \ldots, \mu^{n}, \omega^{1}, \ldots, \omega^{m}\right)$ subject to

$$
\begin{gathered}
x(a)=0=x(b) \\
\theta^{1}\left(\begin{array}{l}
t, x(t), u(t), z(t), \lambda^{1}, \ldots, \lambda^{m}, \\
\omega^{1}, \ldots, \omega^{m}, \mu^{1}, \ldots, \mu^{m}
\end{array}\right)=0, t \in I \\
\theta^{2}\left(t, x(t), u(t), z(t), \lambda^{1}, \ldots, \lambda^{m}, \mu^{1}, \ldots, \mu^{m}\right)=0, t \in I \\
\sum_{i \in I_{\alpha} I} \int^{i}(t)\left(g^{i}(t, x, u)+x(t)^{T} \hat{\omega}^{j}(t)\right) d t \geq 0, \alpha=1,2, \ldots, r \\
\sum_{j \in J} \int_{\alpha} \mu^{j}(t)\left(h^{j}(t, x, u)+\hat{x}^{j}(t)\right) d t \geq 0, \alpha=1,2, \ldots, r \\
\lambda^{i}(t) \geq 0, t \in I, i=1,2, \ldots, m . \\
z(t) \in K, \omega^{i}(t) \in C^{i}, t \in I, j=1,2, \ldots, m
\end{gathered}
$$

where

$$
\begin{gathered}
\theta^{1}=\theta^{1}(.) \\
=f_{x}+\sum_{i=1}^{m} \lambda^{i}(t)\left(g_{x}^{i}(t)+\omega^{i}(t)\right)+\mu(t)^{T} h_{x}+\dot{\mu}(t) \\
\theta^{2}=\theta^{2}(.)=f_{u}+z+\lambda^{T} g_{u}+\mu h_{u}
\end{gathered}
$$

with $\quad f_{x}=f_{x}(t, x(t), u(t)), g_{x}=g_{x}(t, x(t), u(t))$, $h_{x}=h_{x}(t, x(t), u(t))$, etc.

$$
\text { Consider } \theta^{1}\left(\begin{array}{l}
., x(.), u(.), \lambda(.), \mu(.), \\
\dot{\mu}(.), z(.), \omega^{1}(.), \ldots, \omega^{m}(.)
\end{array}\right) \text { as defining a }
$$

mapping $Q^{(1)}: X \times U \times \wedge \times V \times Z \times W \rightarrow B^{1} \quad$ and $\theta^{1}\left(., x(),. u(),. \lambda(),. \mu(),. z(),. \omega^{1}(),. \ldots, \omega^{m}().\right) \quad$ as defining a mapping $Q^{(2)}: X \times U \times \Lambda \times V \times Z \times W \rightarrow B^{2}$ where (i) $B^{1}$ and $B^{2}$ are Banach spaces,

(ii) $\Lambda, V, Z$ and $W$ are spaces of piecewise smooth functions $\lambda, \mu, z$ and $\omega=\left(\omega^{1}, \ldots, \omega^{m}\right)$.

In order to apply the results of [1], some restrictions are required on the equality constraints $\theta^{1}()=$.0 and $\theta^{2}()=$.0 . It suffices if the Fre'che't derivatives $Q^{\prime(1)}=\left(Q_{x}^{1}, Q_{u}^{1}, Q_{\lambda}^{1}, Q_{\mu}^{1}, Q_{z}^{1}\right)$ and $Q^{\prime(2)}=\left(Q_{x}^{2}, Q_{u}^{2}, Q_{\lambda}^{2}, Q_{\mu}^{2}, Q_{z}^{2}\right)$ have weak $*$ closed range. In the following theorem, we write $f=f(t, x, u), g=g(t, x, u)$ and $h=h(t, x, u)$.

Theorem 4 (Converse Duality): Assume that

$\left(\mathbf{C}_{1}\right): f, g$ and $h$ are twice continuously differentiable.

$\left(\mathbf{C}_{2}\right): Q^{\prime}$ and $Q^{\prime 2}$ have weak * closed range.
$\left(\mathrm{C}_{3}\right)$ :

$\int_{I}\left\{f(t, . .)+.() z+.\sum_{i \in I_{0}} \lambda^{i}(t)\left(\begin{array}{l}g^{i}(t, . . .)+(.) \omega^{j} \\ \left.+\sum_{j \in J_{0}} \mu^{j}(t)\left(\begin{array}{c}h^{j}(t, . . .) \\ -\dot{x}^{j}(t)\end{array}\right)\right)\end{array}\right)\right\} d t$ is pseudoconvex.

$\left(\mathbf{C}_{4}\right): \quad \sum_{i \in I_{\alpha} I} \int_{i} \lambda^{i}(t)\left(g^{i}(t, . .)-.(.) \omega^{j}\right) d t . \quad$ and $\sum_{j \in J_{\alpha} I} \int_{I} \mu^{j}(t)\left(h^{j}(t, . .)-.\dot{x}^{j}(t)\right) d t$ are quasiconvex,

(C) $\left.\mathbf{C}_{5}\right) \int_{I} \sigma(t)^{T} A \sigma(t) d t \Rightarrow \sigma(t)=0, t \in I$, where $\sigma(t)$ is an appropriate vector function, and

$$
A=\left(\begin{array}{ll}
f_{x x}+\lambda(t)^{T} g_{x x} & f_{u x}+\lambda(t)^{T} g_{u x} \\
+\mu(t)^{T} h_{x x} & +\mu(t)^{T} h_{u x} \\
f_{x u}+\lambda(t)^{T} g_{x u} & f_{u u}+\lambda(t)^{T} g_{u u} \\
+\mu(t)^{T} h_{x u} & +\mu(t)^{T} h_{u u}
\end{array}\right)
$$

$\left(\mathbf{C}_{6}\right): \mu(a)=\mu(b)$

and

$\left(\mathbf{C}_{7}\right):\left\{\begin{array}{l}\sum_{i \in I_{\alpha}} \lambda^{i}(t)\left(g_{x}^{i}+\omega^{i}\right), \\ \sum_{j \in J \alpha}\left(\mu^{j}(t) h_{x}^{j}+\dot{\mu}^{j}\right) \alpha=1,2, \ldots, r\end{array}\right\}$ are linearly independent.

Then $(\bar{x}, \bar{u})$ is an optimal solution of (CP) and the optimal values of (CP) and (GCD) are equal.

Proof: Since $\left(\bar{x}, \bar{u}, \bar{\lambda}^{1}, \ldots, \bar{\lambda}^{m}, \bar{z}, \bar{\mu}^{1}, \ldots, \bar{\mu}^{m}, \bar{\omega}^{1}, \ldots, \bar{\omega}^{m}\right)$ is an optimal solution of (GCD), by Proposition 1 there exist Langranges multipliers $\tau \in R$, piecewise smooth $\beta: I \rightarrow R^{n}, \quad r: I \rightarrow R^{m}, \quad \eta: I \rightarrow R^{m}, \quad \tau_{\alpha}^{1} \in R \quad$ and $\tau_{\alpha}^{2}, \alpha=1,2, \ldots, r, \tau_{\alpha}^{2}, \alpha=1,2, \ldots, r$ such that

$$
\begin{aligned}
& \tau\left(f_{x}+\sum_{i \in I_{0}} \lambda^{i}(t)\left(g_{x}^{i}+\omega^{i}\right)+\sum_{j \in J 0}\left(\mu^{j}(t) h_{x}^{j}+\dot{\mu}^{j}\right)\right) \\
& +\beta(t)^{T}\left(f_{x x}+\lambda(t)^{T} g_{x x}+\mu(t)^{T} h_{x x}\right) \\
& +r(t)^{T}\left(f_{u x}+\lambda(t)^{T} g_{u x}+\mu(t)^{T} h_{u x}\right) \\
& +\sum_{\alpha=1}^{r} \tau_{\alpha}^{1}\left(\sum_{i \in I_{0}} \lambda^{i}(t)\left(g_{x}^{i}+\omega^{i}\right)\right) \\
& +\sum_{\alpha=1}^{r} \tau_{\alpha}^{2}\left(\sum_{j \in J}\left(\mu^{j}(t) h_{x}^{j}+\dot{\mu}^{j}(t)\right)\right)=0, t \in I \\
& \quad\left(f_{u}+z+\sum_{i \in I_{0}} \lambda^{i}(t) g_{u}^{i}+\sum_{j \in J 0} \mu^{j}(t) h_{u}^{j}\right) \\
& +\beta(t)\left(f_{x u}+\lambda(t)^{T} g_{x u}+\mu(t)^{T} h_{x u}\right)
\end{aligned}
$$




$$
\begin{aligned}
& +r(t)^{T}\left(\begin{array}{l}
f_{u u}+\lambda(t)^{T} g_{u u} \\
+\mu(t)^{T} h_{u u}
\end{array}\right)+\sum_{\alpha=1}^{r} \tau_{\alpha}^{1}\left(\sum_{i \in I_{\alpha}} \lambda^{i}(t) g_{x}^{i}\right) \\
& +\sum_{\alpha=1}^{r} \tau_{\alpha}^{2}\left(\sum_{j \in J_{\alpha}} \mu^{j}(t) h_{u}^{j}\right)=0, t \in I \\
& \tau\left(g^{i}+\bar{x}^{T} \omega^{i}\right)+\beta(t)^{T}\left(g_{X}^{i}+\omega^{i}\right) \\
& +r(t)^{T} g_{u}^{i}+\eta^{i}(t)=0, i \in I_{0}, t \in I \\
& \tau_{\alpha}^{1}\left(g^{i}+\bar{x}^{T} \omega^{i}\right)+\beta(t)^{T}\left(g_{X}^{i}+\omega^{i}\right) \\
& +r(t)^{T} g_{u}^{i}+\eta^{i}(t)=0, i \in I_{\alpha}, \alpha=1,2, . ., r, t \in I \\
& \tau\left(h^{j}-\dot{x}^{j}\right)+\beta(t)^{T} h_{x}^{i}+r(t)^{T} h_{u}^{i}=\dot{\beta}(t), t \in I \\
& \tau_{\alpha}^{2}\left(h^{j}-\dot{x}^{j}\right)+\beta(t) h_{x}^{j}+r(t)^{T} h_{u}^{i}=\dot{\beta}(t), \\
& t \in I, i \in J_{\alpha}, \alpha=1,2, \ldots, r \\
& \tau \lambda^{i}(t) x+\beta(t) \lambda^{i}(t) \in-N_{C}\left(\omega^{i}\right), i \in I_{0} \\
& \beta(t) \lambda^{i}+\tau_{\alpha}^{1} \lambda^{i}(t) x \in-N_{C^{i}}\left(\omega^{i}\right), i \in J_{\alpha}, \alpha=1,2 \ldots, r \\
& \alpha u+r(t) \in-N_{K}(z) \\
& \tau_{\alpha}^{1} \sum_{i \in I_{\alpha}} \int_{I} \lambda^{i}(t)\left(g^{i}+x^{T} \omega^{i}\right) d t=0 \\
& \tau_{\alpha}^{2} \sum_{j \in J_{\alpha} I} \int_{I} \mu^{j}(t)\left(h^{j}+\dot{x}^{j}\right) d t=0 \\
& \eta^{T} \lambda=0 \\
& \left(\tau, \eta(t), \tau_{1}^{1}, \ldots, \tau_{r}^{1}, \tau_{1}^{2}, \ldots, \tau_{r}^{2}\right) \geq 0, t \in I \\
& \left(\tau, \beta(t), r(t), \eta, \tau_{1}^{1}, \ldots, \tau_{r}^{1}, \tau_{1}^{2}, \ldots, \tau_{r}^{2}\right) \neq 0, t \in I
\end{aligned}
$$

Multiplying (21) by $\bar{\lambda}^{i}(t), i \in I_{\alpha}, \alpha=1,2, . ., r$ and using (29), we have

$$
\begin{aligned}
& \tau_{\alpha}^{1} \int_{I} \sum_{i \in I_{\alpha}} \lambda^{i}(t)\left(g^{i}+\omega^{i}\right) d t \\
& +\int_{I} \beta(t)^{T} \sum_{i \in I_{\alpha}} \lambda^{i}(t)\left(g_{X}^{i}+\omega^{i}\right) d t \\
& +\int_{I} r(t)^{T}\left(\sum_{i \in I_{\alpha}} \lambda^{i}(t)^{T}\left(g_{X}^{i}+\omega^{i}\right)\right) d t=0
\end{aligned}
$$

From (27), we have

$$
\int_{I}\left\{\begin{array}{l}
\beta(t)^{T}\left(\sum_{i \in I_{\alpha}} \lambda^{i}(t)\left(g_{x}^{i}+\omega^{i}\right)\right) \\
+r(t)\left(\sum_{i \in I_{\alpha}} \lambda^{i}(t) g_{x}^{i}\right)
\end{array}\right\} d t=0
$$

which can be written as

$$
\int_{I}(\beta(t), r(t))\left(\begin{array}{l}
\sum_{i \in I_{\alpha}} \lambda^{i}(t)\left(g_{x}^{i}+\omega^{i}\right) \\
\sum_{i \in I_{\alpha}} \lambda^{i}(t) g_{X}^{i}
\end{array}\right) d t=0
$$

Multiplying (23) by $\mu^{j}(t), j \in J_{\alpha}, \alpha=1,2, \ldots, r$, we have

$\tau_{\alpha}^{2} \int_{I} \sum_{j \in J_{\alpha}} \mu^{j}(t)\left(h^{j}-\dot{x}^{j}\right) d t+\int_{I}\left\{\begin{array}{l}\beta(t)\left(\sum_{j \in J_{\alpha}} \mu^{j}(t) h_{x}^{i}\right) \\ +\gamma(t)\left(\sum_{j \in J_{\alpha}} \mu^{j}(t) h_{u}^{i}\right)\end{array}\right\} d t$

$=\int_{I} \sum_{j \in J_{\alpha}} \mu^{j}(t) \dot{\beta}(t) d t$.

From (28), we have

$$
\begin{aligned}
& \int_{I}\left\{\beta(t)\left(\sum_{j \in J_{\alpha}} \mu^{j}(t) h_{x}^{i}\right)+\gamma(t)\left(\sum_{j \in J_{\alpha}} \mu^{j}(t) h_{u}^{i}\right)\right\} d t \\
& =\left.\sum_{j \in J_{\alpha}} \mu^{j}(t) \beta(t)\right|_{t=a} ^{t=b}-\sum_{j \in J_{\alpha} I} \int_{I^{j}} \dot{\mu}^{j}(t) \beta(t) d t
\end{aligned}
$$

(by integrating by parts)

which on using the hypothesis $\left(\mathrm{C}_{5}\right)$, given in the relation can be written in the matrix form as

$$
\int_{I}(\beta(t), \gamma(t))\left(\begin{array}{c}
\sum_{j \in J_{\alpha}} \mu^{j}(t) h_{x}^{j}+\mu^{j}(t) \\
\sum_{j \in J_{\alpha}} \mu^{j}(t) h_{u}^{j}
\end{array}\right) d t=0 .
$$

Using the equation constraints of (GCD), in (18) and (19) respectively. We have

$$
\begin{aligned}
& \sum_{\alpha=1}^{r}\left(\tau_{\alpha}^{1}-\tau\right)\left(\sum_{i \in I_{\alpha}} \lambda^{i}(t)\left(g_{x}^{i}+\omega^{i}\right)\right) \\
& +\sum_{\alpha=1}^{r}\left(\tau_{\alpha}^{2}-\tau\right)\left(\sum_{j \in J_{\alpha}}\left(\mu^{j}(t) h_{x}^{j}+\dot{\mu}^{j}(t)\right)\right) \\
& +\beta(t)^{T}\left(f_{x x}+\lambda(t)^{T} g_{x x}+\mu(t)^{T} h_{x x}\right) \\
& +\gamma(t)^{T}\left(f_{u x}+\lambda(t)^{T} g_{u x}+\mu(t)^{T} h_{u x}\right)=0, t \in I
\end{aligned}
$$

and

$$
\begin{aligned}
& \sum_{\alpha=1}^{r}\left(\tau_{\alpha}^{1}-\tau\right)\left(\sum_{i \in I_{\alpha}} \lambda^{i}(t) g_{u}^{i}\right) \\
& +\sum_{\alpha=1}^{r}\left(\tau_{\alpha}^{2}-\tau\right)\left(\sum_{j \in J_{\alpha}} \mu^{j}(t) h_{u}^{j}\right) \\
& +\beta(t)^{T}\left(f_{x u}+\lambda(t)^{T} g_{x u}+\mu(t)^{T} h_{x u}\right) \\
& +\gamma(t)^{T}\left(f_{u u}+\lambda(t)^{T} g_{u u}+\mu(t)^{T} h_{u u}\right)=0, t \in I
\end{aligned}
$$


Combining (34) and (35), we have

$$
\begin{aligned}
& \sum_{\alpha=1}^{r}\left(\tau_{\alpha}^{1}-\tau\right)\left(\begin{array}{c}
\lambda^{i}(t)\left(g_{x}^{i}+\omega^{i}\right) \\
\sum_{i \in I_{\alpha}} \lambda^{i}(t) g_{u}^{i}
\end{array}\right) \\
& +\sum_{\alpha=1}^{r}\left(\tau_{\alpha}^{2}-\tau\right)\left(\begin{array}{c}
\sum_{j \in J_{\alpha}}\left(\mu^{j}(t) h_{x}^{j}+\dot{\mu}^{j}(t)\right) \\
\sum_{j \in J_{\alpha}} \mu^{j}(t) h_{u}^{j}
\end{array}\right) \\
& +\left(\begin{array}{ll}
f_{x x}+\lambda(t)^{T} g_{x x} & f_{u x}+\lambda(t)^{T} g_{u x} \\
+\mu(t)^{T} h_{x x} & +\mu(t)^{T} h_{u x} \\
f_{x u}+\lambda(t)^{T} g_{x u} & f_{u u}+\lambda(t)^{T} g_{u u} \\
+\mu(t)^{T} h_{x u} & +\mu(t)^{T} h_{u u}
\end{array}\right)\left(\begin{array}{c}
\beta(t) \\
\gamma(t)
\end{array}\right)=0 .
\end{aligned}
$$

Ppremultiplying by $(\beta(t), \gamma(t))$, this gives

$$
\begin{aligned}
& \sum_{\alpha=1}^{r}\left(\tau_{\alpha}^{1}-\tau\right) \int_{I}(\beta(t), \gamma(t))\left(\begin{array}{c}
\lambda^{i}(t)\left(g_{x}^{i}+\omega^{i}\right) \\
\sum_{i \in I_{\alpha}} \lambda^{i}(t) g_{u}^{i}
\end{array}\right) d t \\
& +\sum_{\alpha=1}^{r}\left(\tau_{\alpha}^{2}-\tau\right) \int_{I}(\beta(t), \gamma(t))\left(\begin{array}{c}
\sum_{j \in J_{\alpha}}\left(\mu^{j}(t) h_{x}^{j}+\dot{\mu}^{j}(t)\right) \\
\sum_{j \in J_{\alpha}} \mu^{j}(t) h_{u}^{j}
\end{array}\right) d t
\end{aligned}
$$

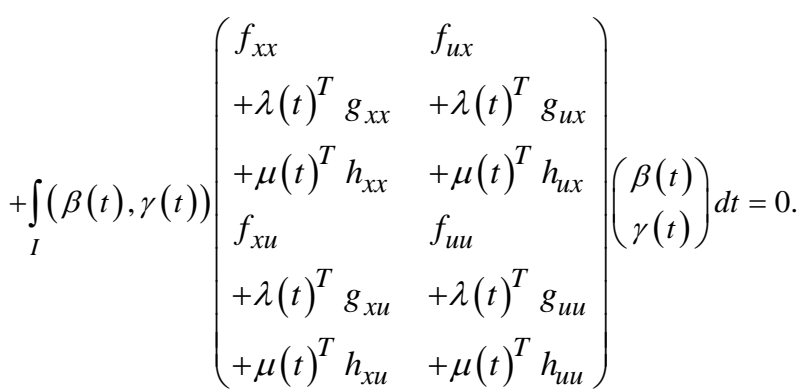

This, on using (32) and (33) gives

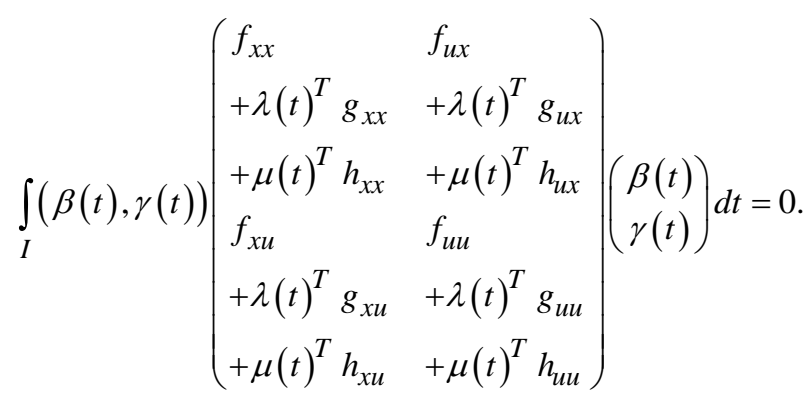

In view of the hypothesis $\left(\mathrm{C}_{4}\right)$, this yields

$$
\beta(t)=0=\gamma(t), t \in I
$$

Using (36), we have

$$
\begin{aligned}
& \sum_{\alpha=1}^{r}\left(\tau_{\alpha}^{1}-\tau\right)\left(\sum_{i \in I_{\alpha}} \lambda^{i}(t)\left(g_{X}^{i}+\omega^{i}\right)\right) \\
& +\sum_{\alpha=1}^{r}\left(\tau_{\alpha}^{2}-\tau\right)\left(\sum_{j \in J_{\alpha}}\left(\mu^{j}(t) h_{X}^{j}+\dot{\mu}^{j}(t)\right)\right)=0 .
\end{aligned}
$$

This because of $\left(\mathrm{C}_{6}\right)$, yields

$$
\begin{aligned}
& \tau_{\alpha}^{1}-\tau=0, \tau_{\alpha}^{2}-\tau=0, \\
& \alpha=1,2, \ldots, r
\end{aligned}
$$

If $\tau=0$, then $\tau_{\alpha}^{1}=0=\tau_{\alpha}^{2}, \alpha=1,2, \ldots, r$. and from (20) and (21), we have $\eta(t)=0, t \in I$.

$$
\text { Consequently, we have }\left(\begin{array}{l}
\tau, \tau_{1}^{1}, \ldots, \tau_{r}^{1}, \tau_{1}^{2}, \ldots, \tau_{r}^{2}, \\
\beta(t), \gamma(t), \eta(t)
\end{array}\right)=0 \text {, }
$$

$t \in I$ ensuing a contradiction to (31).

Hence $\tau>0$, implying $\tau_{\alpha}^{1}>0, \tau_{\alpha}^{2}>0, \alpha=1,2, \ldots, r$. From (20) together with (21) and (22) together with (23), we have

$$
\begin{gathered}
g_{x}^{i}+\bar{x}^{T} \bar{\omega}^{i} \leq 0, t \in I \\
\lambda^{i}\left(g_{x}^{i}+\bar{x}^{T} \bar{\omega}^{i}\right)=0, i \in I_{0} \\
h^{i}-\dot{\bar{x}}^{i}=0, t \in I \\
\mu^{j}(t)\left(h^{j}-\dot{\bar{x}}^{j}\right)=0, t \in I, j \in J_{0}
\end{gathered}
$$

The relations (24)-(26), we have

$$
\begin{gathered}
\bar{x}(t)^{T} \omega^{j}(t)=S\left(\bar{x}(t) \mid C^{j}\right), j=1,2, \ldots, m \\
\bar{u}(t)^{T} z(t)=S(\bar{u} \mid K)
\end{gathered}
$$

From (37), (39) and (41), we have

$$
g^{i}(t, \bar{x}, \bar{u})+S\left(\bar{x}(t) \mid C^{i}\right) \leq 0, i=1,2, \ldots, m
$$

and

$$
h(t, \bar{x}, \bar{u})-\dot{\bar{x}}=0 .
$$

implying that $(\bar{x}, \bar{u})$ is feasible for (CP).

From (38), (40) and (42), we have

$$
\begin{aligned}
& \int_{I}(f(t, \bar{x}, \bar{u})+S(u(t) \mid K)) d t \\
& =\int_{I}\left\{\begin{array}{l}
f(t, \bar{x}, \bar{u})+\bar{u}(t)^{T} z(t) \\
+\sum_{i \in I_{0}} \lambda^{i}(t)\left(g^{i}(t, \bar{x}, \bar{u})+\bar{x}^{T} \bar{\omega}^{i}(t)\right) \\
+\sum_{j \in J_{0}} \mu^{j}(t)\left(h^{j}(t, \bar{x}, \bar{u})-\dot{\bar{x}}^{j}\right)
\end{array}\right\} d t
\end{aligned}
$$

In view of the hypotheses $\left(\mathrm{C}_{3}\right)$ and $\left(\mathrm{C}_{4}\right)$, by Theorem 1 , the optimality of $(\bar{x}, \bar{u})$ for (CP) follows.

\section{Special Cases}

If $I_{0}=M$ and $J_{0}=N$, then (GCD) becomes (WCD) which is Wolfe type dual to (CD) under the pseudoconvexity of 


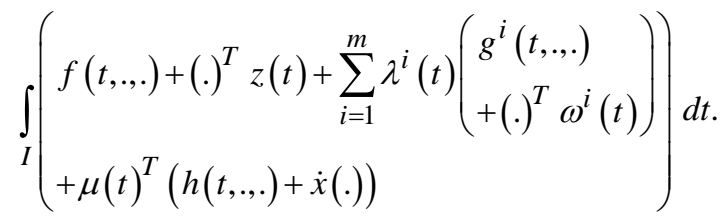

If $I_{0}=\phi$ and $I_{\alpha}=M$, (for some $\alpha \in\{1,2, \ldots, r\}$ ), then (GCD) becomes (M-WCD) is a Mond-Weir type dual to (CP) if $\int_{I}\left(f(t, . .)+.(.)^{T} z(t)\right) d t$ is pseudoconvex, and $\int_{I} \sum_{i=1}^{m} \lambda^{i}(t)\left(\begin{array}{l}g^{i}(t, . . .) \\ +(.)^{T} \omega^{i}(t)\end{array}\right) d t$ and $\int_{I} \mu(t)^{T}\left(\begin{array}{c}h(t, . . .) \\ +\dot{x}(t)\end{array}\right) d t$ are quasiconvex.

Let $B(t)$ and $D^{j}(t),(j=1,2, \ldots, m)$ be positive semi definite matrices and continuous on $I$. Then $S(u(t) \mid K)=\left(x(t)^{T} B(t) x(t)\right)^{\frac{1}{2}}, t \in I \quad$ where $K=\left\{B(t) z(t) \mid z(t)^{T} B(t) z(t) \leq 1, t \in I\right\} \quad$ and $S\left(x(t) \mid C^{j}\right)=\left(x(t)^{T} D^{j}(t) x(t)\right)^{1 / 2}, t \in I, j=1,2, \ldots, m$ where $C^{j}=\left\{D^{j}(t) \omega^{j}(t) \mid \omega^{j}(t)^{T} D^{j}(t) \omega^{j}(t) \leq 1, t \in I\right\}$.

Replacing the support function by its corresponding square root of a quadratic form, we have $\left(\mathbf{C P}_{\mathbf{0}}\right)$ : Minimize $\int_{I}\{f(t, x, u)+S(u(t) \mid K)\} d t$ subject to

$$
\begin{gathered}
x(a)=0=x(b) \\
g^{j}(t, x, u)+\left(x(t)^{T} D^{j}(t) x(t)\right)^{1 / 2} \leq 0, t \in I, j=1,2, \ldots, m
\end{gathered}
$$$$
h(t, x, u)=\dot{x}(t), t \in I
$$$$
\left.\mathbf{( G C D}_{0}\right) \text { : Maximize } \int_{I}\left\{\begin{array}{l}
f(t, x, u)+u(t)^{T} B(t) z(t) \\
+\sum_{i \in I_{0}} \lambda^{i}(t)\left(\begin{array}{l}
g^{i}(t, x, u) \\
+x(t)^{T} D^{i}(t) \omega^{i}(t)
\end{array}\right) \\
+\sum_{j \in J_{0}} \mu^{j}(t)\left(h^{j}(t, x, u)-\dot{x}^{j}(t)\right)
\end{array}\right) d t
$$

subject to

$$
\begin{gathered}
x(a)=0=x(b) \\
f_{x}(t, x, u)+B(t) z(t)+\sum_{i=1}^{m} \lambda^{i}(t)\left(\begin{array}{l}
g_{x}^{i}(t, x, u) \\
+D^{i}(t) \omega^{i}(t)
\end{array}\right) \\
+\mu(t)^{T} h_{x}=\dot{\mu}(t), t \in I \\
f_{u}(t, x, u)+B(t) z(t)+\lambda(t)^{T} g_{u}(t, x, u) \\
+\mu(t)^{T} h_{u}(t, x, u)=0, t \in I \\
z(t)^{T} B(t) z(t) \leq 1, t \in I
\end{gathered}
$$

$$
\begin{gathered}
\omega^{j}(t) D^{j}(t) \omega^{j}(t) \leq 1, t \in I, j=1,2, \ldots, m \\
\sum_{i \in I_{\alpha}} \int_{I} \lambda^{i}(t)\left(g^{i}(t, x, u)+D^{i}(t) x(t)\right) d t \geq 0, \alpha=1,2, \ldots, r \\
\sum_{j \in I_{\alpha}} \mu^{j}(t)\left(h^{j}(t, ., u)-x^{j}(t)\right) d t \geq 0, \alpha=1,2, \ldots, r \\
\lambda^{i}(t) \geq 0, t \in I, i=1,2, . ., m,
\end{gathered}
$$

These dual models are not explicitly reported in the literature. However, the duality relationship between $\left(\mathrm{CD}_{0}\right)$ and $\left(\mathrm{GCD}_{0}\right)$ can be established analogously to that of the problem of preceding section.

\section{Nonlinear Programming Problem}

If all the functions involved in the formulation of (CP) and (GCD) are independent of $t$, these problem reduce to the following nonlinear programming problems with support functions which do not appear in the literature.

(NP): Minimize $f(x, u)+S(u \mid K)$

subject to

$$
\begin{gathered}
g^{j}(x, u)+S\left(x \mid C^{j}\right) \leq 0, j=1,2, \ldots, m \\
h(x, u)=0 \\
f_{x}(x, u)+u^{T} z
\end{gathered}
$$

(GND): Maximize $+\sum_{i \in I_{0}} \lambda^{i}\left(g^{i}(x, u)+x^{T} \omega^{i}\right)$

$$
+\sum_{J \in j_{0}} \mu^{T} h^{j}(x, u)
$$

subject to

$$
\begin{gathered}
f_{x}(x, u)+\sum_{i=1}^{m} \lambda^{i}\left(g_{x}^{i}(x, u)+\omega^{i}\right)+\mu^{T} h_{x}(x, u)=0 \\
f_{u}(x . u)+z+\lambda^{T} g_{u}(x . u)+\mu^{T} h_{u}(x, u)=0, \\
z \in K \text { and } \omega^{j} \in C^{j}, j=1,2, \ldots, m \\
\sum_{i \in I_{\alpha}} \lambda^{i}\left(g^{i}(x, u)+x^{T} \omega^{i}\right) \geq 0, \alpha=1,2, \ldots, \mathrm{r} \\
\sum_{J \in j_{\alpha}} \mu^{j} h^{j}(x, u) \geq 0, \alpha=1,2, \ldots, \mathrm{r} \\
\lambda^{i}(t) \geq 0,, t \in I, i=1,2, \ldots, m
\end{gathered}
$$

Ignoring $h(x, u)$ and replacing $f(x, u), g(x, u)$ and $s(u \mid K)$ by $f(x), g(x)$ and $s(x \mid K)$ respectively, we get the following problems studied by Husain and Jabeen [2]:

Primal $\left(\mathbf{P}_{\mathbf{1}}\right)$ : Minimize $f(x)+s(x \mid K)$

subject to

$$
\begin{gathered}
g^{i}(x)+s\left(x \mid C^{i}\right) \leq 0, i=1,2, \ldots, m \\
h(x)=0
\end{gathered}
$$




$$
f(x)+x^{T} z
$$$$
z \in K, \omega^{i} \in C^{i}, i=1,2, \ldots, m .
$$

Dual (GD): Maximize $+\sum_{i \in I_{0}} \lambda^{i}\left(g^{i}+x^{T} \omega^{i}\right)$

$$
+\sum_{j \in J_{0}} \mu^{j} h^{j}(x)
$$

subject to

$$
\begin{gathered}
f_{x}(x)+z+\sum_{i=1}^{m} \lambda^{T}\left(g_{x}^{i}+\omega^{i}\right)=0 \\
\sum_{i \in I_{\alpha}}^{m} \lambda^{i}\left(g^{i}+x^{T} \omega^{i}\right) \geq 0, \alpha=1,2, \ldots, r \\
\sum_{j \in J_{\alpha}} \mu^{j}(t)^{T} h^{j}(x) \geq 0 \alpha=1,2, \ldots, r
\end{gathered}
$$

\section{References}

[1] S. Chandra, B.D. Craven, and I. Husain, 'A class of nondifferential Control problems', J. Optim. Theory Appl. 56 (1988), 227-243.

[2] I. Husain and Z. Jabeen, 'Mixed duality for a Programming containing support functions', J. Appl. Math \& computing Vol. 15 (2004), No 1-2 pp. 211-225.

[3] I. Husain, A. Ahmad and Abdul Raoof Shah, 'On a control problem with support functions', submitted for publication.

[4] I. Husain, Abdul Raoof Shah and Rishi K. Pandey, 'Duality for a control Problem with support function', submitted for publication.

[5] B. Mond and M. Hanson, 'Duality for control problem', SIAM J. Control 6 (1968), 114-120. 